\title{
Near-field Propagation in Planar Nanostructured Arrays
}

\author{
David L. Andrews and Richard G. Crisp \\ Nanostructures and Photomolecular Systems, School of Chemical Sciences and Pharmacy, \\ University of East Anglia, Norwich NR4 7TJ, UK
}

\begin{abstract}
In suitably designed nanoscale systems the ultrafast migration of uv/visible electromagnetic energy, despite its nearfield rather than propagating character, can be made highly directional. At the photon level such energy migration generally takes a multi-step form, with each step signifying the transfer of an electromagnetic quantum between chromophores playing the transient roles of source/donor and detector/acceptor. There is much interest in nanophotonic devices based on such mechanisms, although the excitation transfer is usually subject to losses such as radiative decay, and possible device applications are compromised by a lack of suitable control mechanisms. Until recently it appeared that only by inefficient and kinetically frustrated means, such as chromophore reorientation or movement, could significant control be effected. However in a system constructed to inhibit near-field propagation by geometric configuration, the throughput of laser pulses can facilitate energy transfer through a process of laser-assisted resonant energy transfer. Suitably configuring an arrangement of dipoles, it proves possible to design parallel arrays of optical donors and acceptors such that the transfer of energy from any single donor, to its counterpart in the opposing plane, is switched by throughput laser radiation of an appropriate intensity, frequency and polarization. A detailed appraisal of some possible realizations of this system reveals an intricate interplay of electronic structure, optical frequency and geometric factors. In the drive to miniaturize ultrafast optical switching and interconnect devices, the results suggest a new basis for optically activated transistor action in nanoscale components, with significant parallel processing capability.
\end{abstract}

Keywords: Near-field, energy transfer, quantum electrodynamics, optical switching, optical communications, nanoarrays

\section{INTRODUCTION}

In heterogeneous, optically complex systems, any primary excitation produced on the absorption of uv/visible light by a local optical center or chromophore will generally be followed by a process of relaxation back down to the ground state, with an associated redistribution of the excitation energy on a timescale determined by the lifetime of the electronic excited state. While this relaxation may take the form of fluorescence propagating out of the system, it is often a process that entails a series of ultrafast excitation transfers between closely neighboring optical centers - the mechanism for each step involving near-field electromagnetic coupling. At the photon level each of these steps signifies the transfer of an electromagnetic quantum between chromophores which play the transient roles of source/donor and detector/acceptor. The ability to direct this close-range form of energy transfer is extensively utilized in natural systems, such as the light-harvesting complexes of photosynthetic organisms. ${ }^{1-4}$ There is much interest in nanophotonic systems based on such principles, although possible device applications are frequently compromised by a lack of suitable control mechanisms. Of the many methods available to direct near-field energy transfer, one of the simplest is the application of intense optical radiation to enhance the coupling between chromophores. Although it has only recently begun to attract interest, this mechanism clearly offers a means to exert significant control. This paper develops a concept for its exploitation in the design and operation of an all-optical transistor.

\section{UNPERTURBED ENERGY TRANSFER BETWEEN ARRAYS}

The mechanism for energy transfer from an excited chromophore to another in its ground state, via a process known as resonance energy transfer (RET), is easily calculated using a quantum electrodynamical approach. ${ }^{5-7}$ From second-order perturbation theory, utilizing the Fermi golden rule, the probability of energy transfer is given by: 


$$
P=\frac{2 \pi \rho_{f} t}{\hbar}\left|\sum_{R} \frac{\left\langle F\left|H_{\mathrm{int}}\right| R\right\rangle\left\langle R\left|H_{\mathrm{int}}\right| I\right\rangle}{E_{I}-E_{R}}\right|^{2},
$$

where $F, R$ and $I$ refer to system states - that is, the state of both the chromophores and the radiation field. Also $\rho_{f}$ is the density of final states, and $H_{\text {int }}$ is the interaction Hamiltonian defined as;

$$
H_{\mathrm{int}}=\sum_{\xi}-\varepsilon_{0}^{-1} \boldsymbol{\mu}(\xi) \cdot \boldsymbol{d}^{\perp}\left(R_{\xi}\right)
$$

in which $\boldsymbol{\mu}$ is the electric dipole operator and $\boldsymbol{d}^{\perp}$ is the transverse electric displacement field operator at the specified location;

$$
\boldsymbol{d}^{\perp}\left(R_{\xi}\right)=i \sum_{k, \lambda}\left(\frac{\hbar c k \varepsilon_{0}}{2 V}\right)^{1 / 2}\left\{\boldsymbol{e} \cdot a \cdot e^{i \boldsymbol{k} \cdot \boldsymbol{R}_{\xi}}-\overline{\boldsymbol{e}} \cdot a^{\dagger} \cdot e^{-i \boldsymbol{k} \cdot \boldsymbol{R}_{\xi}}\right\}
$$

The above operator effects the annihilation and creation of photons at the optical centers through the action of the lowering and raising operators $a$ and $a^{\dagger}$, respectively. ${ }^{8}$ Two distinct time-orderings contribute to the result delivered by equation (1), and these are represented by the state-sequence diagrams ${ }^{9}$ given in figure 1.

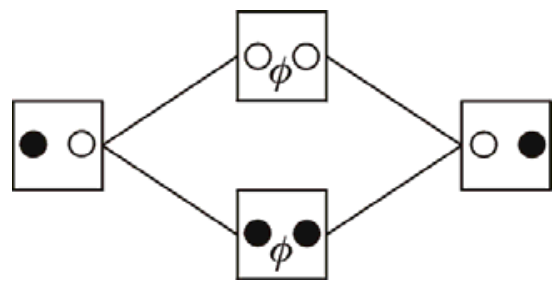

Figure 1: State-sequence diagram for resonance energy transfer. Black species represent an excited state, white a ground state. Also $\phi$ denotes a virtual photon responsible for energy transfer. With the leftmost box denoting the initial state and right the final state, each pathway across the diagram represents one timeordering.

The probability of energy transfer occurring can be calculated from equation (1), and following its full development the result emerges as; ${ }^{5}$

$$
P=\left(\frac{\left|\mu_{D}^{0 e}\right|^{2}\left|\mu_{A}^{e 0}\right|^{2} \rho_{f} t}{8 \pi \hbar \varepsilon_{0}^{2}}\right) \frac{\kappa^{2}}{R^{6}}
$$

where $\mu_{\xi}^{f i}$ is the dipole-transition moment of chromophore $\xi$ for the transition from state $i$ to state $f$ ( 0 indicating the electronic ground state and $e$ a generic excited state), and $R$ is the separation of the chromophores. Additionally, $\kappa$ is a factor dependent on the relative orientations of the transition moments, given as follows;

$$
\kappa=\hat{\boldsymbol{\mu}}_{D}^{0 e} \cdot \hat{\boldsymbol{\mu}}_{A}^{e 0}-3\left(\hat{\boldsymbol{\mu}}_{D}^{0 e} \cdot \hat{\boldsymbol{R}}\right)\left(\hat{\boldsymbol{\mu}}_{A}^{e 0} \cdot \hat{\boldsymbol{R}}\right)
$$

As can be seen, the probability of transfer depends on both the separation and orientation of the transfer pair. Equation (4) is the short-range limit of a more general result for a transfer mechanism that is valid over all distances - in the long range (i.e. at distances large compared to the wavelength for a photon delivering the transfer energy) that mechanism is dominated by the well known inverse-square dependence on the separation of the optical centers, in other words the electromagnetic coupling assumes a fully propagating character. Here we are concerned with chromophore separations of a much smaller magnitude - typically nanoscale. Indeed, this is the type of transfer first described by Förster as radiationless. ${ }^{10}$ It has since been explained by quantum electrodynamics (QED) that the energy transfer is mediated by a virtual photon, ${ }^{11}$ a particle that cannot be detected directly, but which electromagnetically couples the donor relaxation 
and acceptor excitation events through its creation and subsequent annihilation. Due to time-energy uncertainty, the very short timescale over which the transfer mechanism occurs means that energy need not be conserved during the process, provided it is conserved between the initial and final state of the overall transfer. This means that, while the classically obvious pathway of emission by the donor and subsequent absorption by the acceptor is a major contributor to the overall probability of transfer, an equally valid contribution is provided by acceptor emission and subsequent absorption by the donor. Returning to figure 1, it can be seen that the former is represented in the upper pathway, while the other is seen in the lower pathway. Only by adding the contributions from both pathways can one arrive at the full result for RET, as given in eqn (4).

The system envisaged consists of two parallel, planar arrays of chromophores. The first array is uniformly constructed from donor chromophores, the second from acceptor chromophores. Although the donor and acceptor chromophores need not be electronically identical, it is required that at least one electronic transition in the donor overlaps energetically with one in the acceptor. Furthermore, both arrays share the same lattice spacing $l$, and they are positioned one above the other such that each donor is directly above an acceptor. The separation of the two arrays $r$ is necessarily much smaller than the lattice spacing, as will be seen below. Also, the chromophores are oriented such that the principle dipole-transition moments of all the donor chromophores are parallel to each other, but orthogonal to those of the acceptors. A diagram of this setup is given in figure 2. The system is assigned a set of Cartesian axes, where the

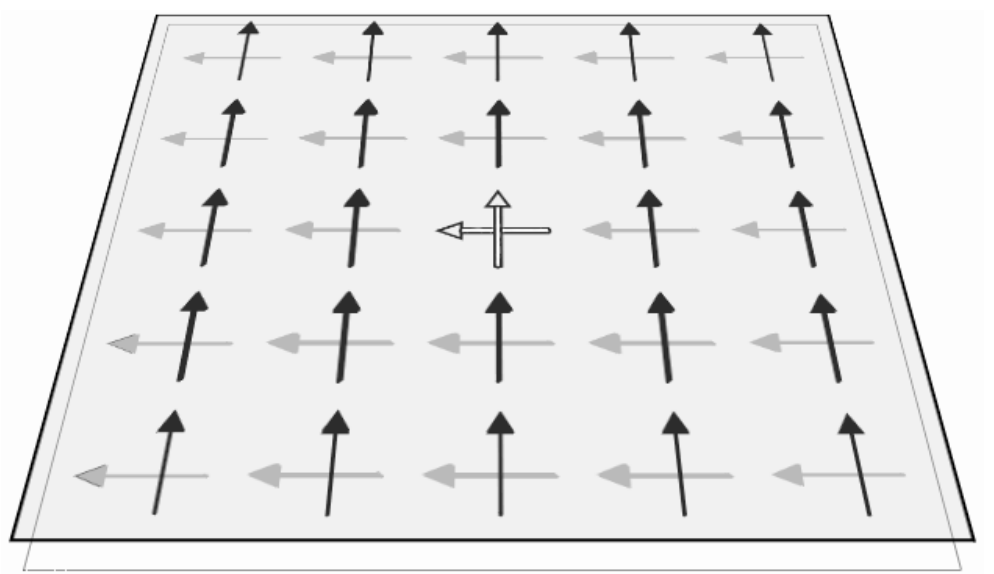

Figure 2: Structure of the array, viewed from above. Both arrays lie in the ij-plane, with all donor transition moments (black) in the upper array parallel to the $i$-axis, and all acceptor transition moments (gray) in the lower array parallel to the $j$ axis. The open arrows represent one excited donor and its counterpart acceptor. Each donor and acceptor chromophore is separated from its nearest neighbor (of the respective type) a distance equal to the lattice spacing $l$. Each donor is a distance $r$ above its corresponding acceptor.

donor transition moments lie along the $i$-axis, the $j$-axis is parallel to the acceptor transition moments and the $z$-axis is perpendicular to the plane of the arrays. The position of each donor from an origin (located on one of the donors) is $u^{\prime} \hat{\mathbf{i}}+v^{\prime} \hat{\boldsymbol{j}}$, where $u^{\prime}$ and $v^{\prime}$ are integers; the position of each acceptor is $u \hat{\boldsymbol{i}}+v \hat{\boldsymbol{j}}+r \hat{\mathbf{k}}$. Introducing;

$$
\hat{\boldsymbol{\mu}}_{D}^{0 e}=\hat{\boldsymbol{i}} \quad ; \quad \hat{\boldsymbol{\mu}}_{A}^{e 0}=\hat{\boldsymbol{j}} \quad ; \quad \boldsymbol{R}=\left(u-u^{\prime}\right) l \hat{\boldsymbol{i}}+\left(v-v^{\prime}\right) \hat{\boldsymbol{j}}+r \hat{\boldsymbol{k}} \quad,
$$

the probability of energy transfer from a given donor to an acceptor is expressible as;

$$
P_{u v}=K t \frac{\kappa^{2}}{\left(\left(u-u^{\prime}\right)^{2}+\left(v-v^{\prime}\right)^{2}+r^{\prime 2}\right)^{3}}=\left(\frac{\left|\mu_{D}^{0 \varepsilon}\right|^{2}\left|\mu_{A}^{\varepsilon 0}\right|^{2} \rho_{f} t}{8 \pi \hbar \varepsilon_{0}^{2} l^{6}}\right) \frac{9\left(u-u^{\prime}\right)^{2}\left(v-v^{\prime}\right)^{2}}{\left(\left(u-u^{\prime}\right)^{2}+\left(v-v^{\prime}\right)^{2}+r^{\prime 2}\right)^{5}},
$$

where $r^{\prime}=r / l$ is the aspect ratio of the system. It is evident from the above equation that if any donor and acceptor share the same column or row in the array (i.e. if $u=u^{\prime}$ or $v=v^{\prime}$ ), then there is a zero probability of energy transfer. Thus, the only viable targets for energy transfer are those acceptors with a relatively large separation from the donor, and activating the energy transfer pathway between a donor and its counterpart acceptor would provide an effective means for directing excitation energy in a predictable direction. 


\section{ACTIVATING ENERGY TRANSFER WITH OPTICAL RADIATION}

A throughput beam of laser radiation can be forward-Rayleigh scattered by any pair of chromophores, enhancing or inhibiting the transfer of energy between them. ${ }^{12}$ In cases where transfer would otherwise be forbidden on geometric grounds, i.e. where the orientation factor $\kappa=0$ - see equations (4), (5) - then LARET (laser-assisted resonance energy transfer) can provide an alternative pathway to enable the process. In the simplest scheme, one photon from the applied radiation mode is absorbed, and an identical photon is emitted by a chromophore pair. With the energy transfer steps also included, the process thus involves four elementary matter-radiation interactions. (Mechanisms involving the absorption and emission of more than one laser photon also contribute to the process, but with a much smaller magnitude; they can safely be discounted for present purposes.) Four distinct channels are possible, differing in the interactions of the applied radiation field with each chromophore; one representative time-ordered diagram for each of these channels is exhibited in figure 3.
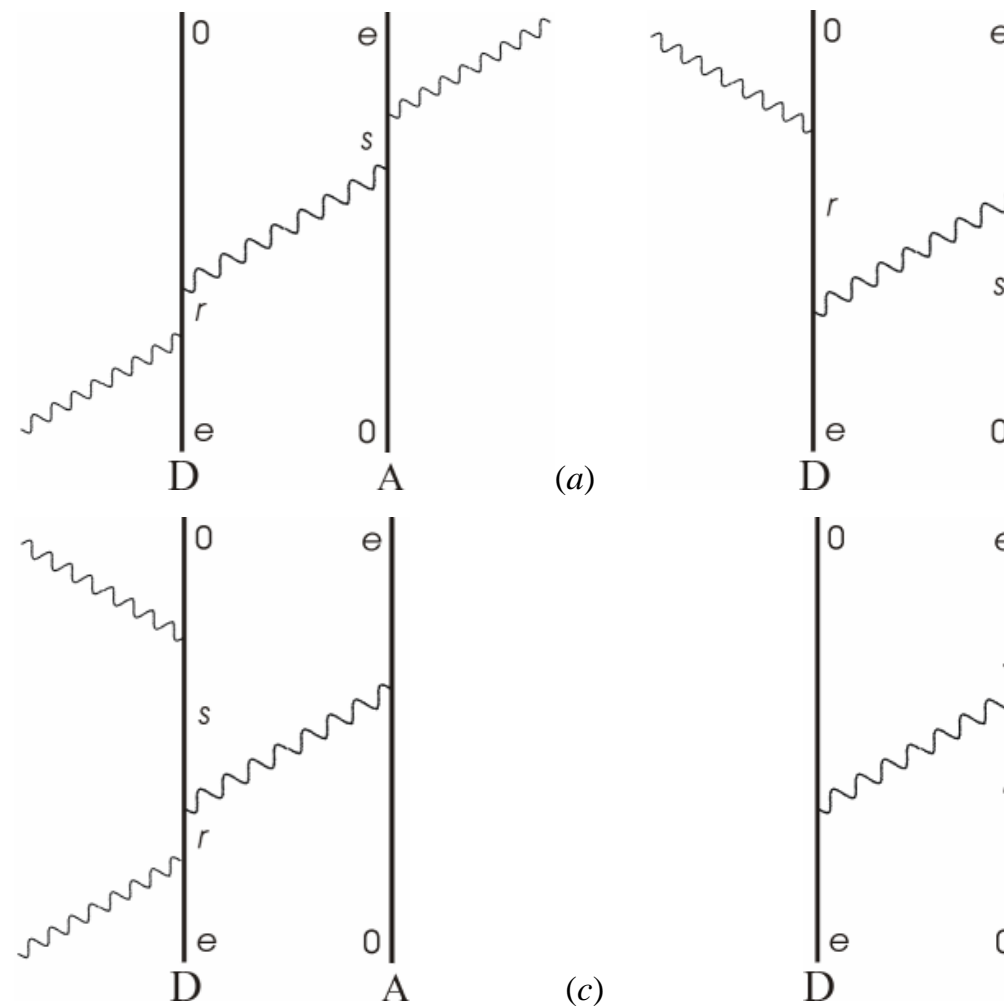

(a)

$\mathrm{D}$

(b)

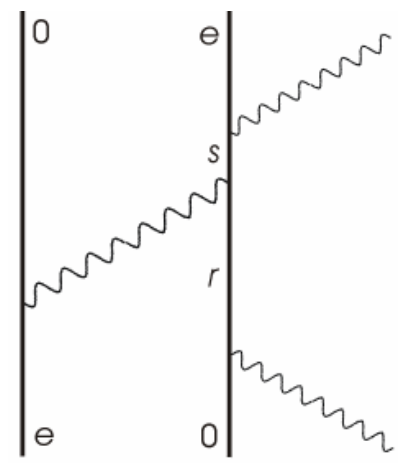

$(c)$

$\mathrm{D}$

A

(d)

Figure 3: Time-ordered diagrams representing the four quantum channels associated with LARET. In the upper two, the laser radiation interacts with both chromophores; the lower two involve laser interactions at only one chromophore. Each channel requires a further 23 permutations of the displayed time-orderings for its full representation.

The probability for energy to transfer between the chromophores via a LARET pathway is given by;

$$
P=\frac{\rho_{f} \int_{0}^{t} I^{2}(t) \mathrm{d} t}{32 \hbar c^{2} \pi \varepsilon_{0}^{4} R^{6}}\left|a^{\prime}+a^{\prime \prime}+b^{\prime}+b^{\prime \prime}\right|^{2},
$$

where the integral of $I^{2}(t)$ describes the irradiance of the laser pulse, evaluated at any instance in time. The four terms on the right-hand side of eqn (8) each describe one quantum channel; the first two involve a single laser-matter interaction on each chromophore; 


$$
\begin{aligned}
& a^{\prime}=\left\{e_{i} \alpha_{i j}^{D}\left(-k^{\prime}\right)\left(\delta_{j k}-3 \hat{R}_{j} \hat{R}_{k}\right) \alpha_{l k}^{A}\left(k^{\prime}\right) \bar{e}_{l}\right\}, \\
& a^{\prime \prime}=\left\{\bar{e}_{i} \alpha_{i j}^{D}\left(k^{\prime}\right)\left(\delta_{j k}-3 \hat{R}_{j} \hat{R}_{k}\right) \alpha_{l k}^{A}\left(-k^{\prime}\right) e_{l}\right\} .
\end{aligned}
$$

Here $\boldsymbol{e}^{\lambda}(k)$ is the polarization vector of the laser pulse (allowed to be complex to account for both circular and planar polarizations; the overbar signifies a complex conjugate). Also, $\alpha_{i j}^{f(\xi)}\left( \pm k^{\prime}\right)$ is the dynamic polarizability of chromophore $\xi$ defined as;

$$
\alpha_{i j}^{f(\xi)}\left( \pm k^{\prime}\right)=\sum_{r}\left[\frac{\mu_{i}^{f r} \mu_{j}^{r i}}{E_{f r} \pm \hbar c k^{\prime}}+\frac{\mu_{j}^{f r} \mu_{i}^{r i}}{E_{i r} \mp \hbar c k^{\prime}}\right] .
$$

The other two terms in eqn (8) are associated with channels in which both laser-matter interactions occur at the same chromophore;

$$
\begin{aligned}
& b^{\prime}=\left\{e_{i} \bar{e}_{j} \beta_{i j k}^{D}\left(\delta_{k l}-3 \hat{R}_{k} \hat{R}_{l}\right) \mu_{l}^{\beta 0(A)}\right\}, \\
& b^{\prime \prime}=\left\{\mu_{i}^{0 e(D)}\left(\delta_{i j}-3 \hat{R}_{i} \hat{R}_{j}\right) \beta_{j k l}^{A} e_{k} \bar{e}_{l}\right\},
\end{aligned}
$$

where $\beta_{i j k}^{\xi}$ is the hyperpolarisability of chromophore $\xi$ - representing a three-quantum interaction. Unless the chromophores are both polar and chiral, the hyperpolarisability is zero and the terms in eqns (11) can be discounted.

Traditionally, the next step in calculating the probability of energy transfer would often involve the application of a two-level approximation, as is common in dealing with many high-order optical processes, basically assuming that any other energy levels within the chromophores are too far removed from the ground and first excited state to contribute to the mechanism in any meaningful way. The structure of the array, however, has been designed specifically to preclude direct energy transfer involving transitions between the donor and acceptor first excited states and the corresponding electronic ground states. Therefore, we are required to consider a third energy level in the scheme for the donor, and equally for acceptor. Although each $\alpha$ tensor contains an intermediate state sum which is effected over all electronic states of the specific species involved, generally one summand is significantly larger in magnitude than the others, because its denominator is small. This can be exploited by selection of a frequency for the applied radiation that has a small resonance offset, such that $E_{\sigma e}=\hbar c k+\Delta E_{D}$, where $\sigma$ is one specific state of D (distinct from $e$ and 0 ) with non-zero transition moments to both $e$ and $0 ; \Delta E_{D}$ is a non-zero energy with significantly lower magnitude than a typical transition energy. An equivalent near-resonant state $\tau$ can be assumed for the acceptors, although $\Delta E_{D}$ and $\Delta E_{A}$ are not required to be equal. Applying these conditions to the polarizabilities contained within equation (9), it becomes apparent that one term for each chromophore will vastly exceed all other terms (as such, all other terms can be discounted as small corrections). Both are obtained from eqn (9a) and are as follows;

$$
\alpha_{i j}^{0 e(D)} \approx \frac{\mu_{j}^{0 \sigma} \mu_{i}^{\sigma e}}{-\Delta E_{D}} ; \alpha_{l k}^{e 0(A)} \approx \frac{\mu_{l}^{e \tau} \mu_{k}^{\tau 0}}{-\Delta E_{A}} .
$$

Both $\boldsymbol{\mu}^{\sigma e}$ and $\boldsymbol{\mu}^{e \tau}$ are linked to the polarization vector of the laser beam. Assuming they are parallel, and that plane polarized light is used (i.e. $\boldsymbol{e}=\overline{\boldsymbol{e}}$ ), the beam can be oriented such that $\boldsymbol{e}$ is parallel to the former two vectors, an easy task given that the array is fixed in a known position. Furthermore, selecting suitable chromophores such that $\boldsymbol{\mu}^{0 \sigma(D)}$ and $\boldsymbol{\mu}^{\tau(A)}$ are orthogonal to the corresponding $\boldsymbol{\mu}^{0 e(D)}$ and $\boldsymbol{\mu}^{e 0(A)}$ transition moments, and parallel to $\hat{\boldsymbol{k}}$, the system now possesses the opportunity for direct transfer between a donor and its corresponding acceptor, on the application of laser light, effectively creating an externally switchable energy transfer system. 
In order to consider device applications, it must be ensured that the probability of energy transfer to the intended acceptor is much greater than that to any other acceptor. To this end, the probability of energy transfer from an excited donor situated at the origin to every acceptor in the array will be calculated below. As seen in the previous section, there is a zero probability of RET transfer to the intended acceptor, however both RET and LARET present pathways for energy flow to other acceptors in the array. Thus, the total probability must be calculated by combining the probability of both processes. This leads to a total of three terms; two are the probabilities of RET and LARET, as given earlier by eqns (4) and (8). The final term is the quantum interference between the two pathways, given by;

$$
P=\frac{\left|\mu_{A}^{0 \alpha}\right|\left|\mu_{B}^{\beta 0}\right| \kappa \rho_{f} \int_{0}^{t} I(t) \mathrm{d} t}{8 \hbar c \pi \varepsilon_{0}^{3} R^{6}} \mathfrak{R e}\left\{a^{\prime}+a^{\prime \prime}+b^{\prime}+b^{\prime \prime}\right\}
$$

Applying the conditions described above, a second orientation factor can be defined, describing the orientation of the transition moments involved in LARET;

$$
\kappa^{\prime}(u, v)=\frac{u^{2}+v^{2}-2 r^{\prime 2}}{u^{2}+v^{2}+r^{\prime 2}}
$$

allowing the total probability of energy transfer from the excited donor to any given acceptor $(u, v)$ to be expressed as;

$$
P_{u v}(t)=K \times \frac{9 u^{2} v^{2}-6 \operatorname{Cuv}\left(u^{2}+v^{2}-2{r^{\prime 2}}^{2}\right) \int_{0}^{t} I(t) d t+C^{2}\left(u^{2}+v^{2}-2{r^{\prime}}^{2}\right)^{2} \int_{0}^{t} I^{2}(t) d t}{\left(u^{2}+v^{2}+r^{\prime 2}\right)^{5}} .
$$

As opposed to the former, unirradiated system, now energy transfer is possible to any acceptor within the array. Detailed calculations to determine the optimum setup for the array have been performed elsewhere; ${ }^{13}$ it transpires that, with an irradiance of $10^{14} \mathrm{~W} \mathrm{~m}^{-2}$ and an aspect ratio of $1: 20$, irradiating the array activates a definite pathway for transfer to the intended acceptor, without noticeably influencing the probability of transfer to an undesirable target. Graphs showing this effect are shown in figure 4.
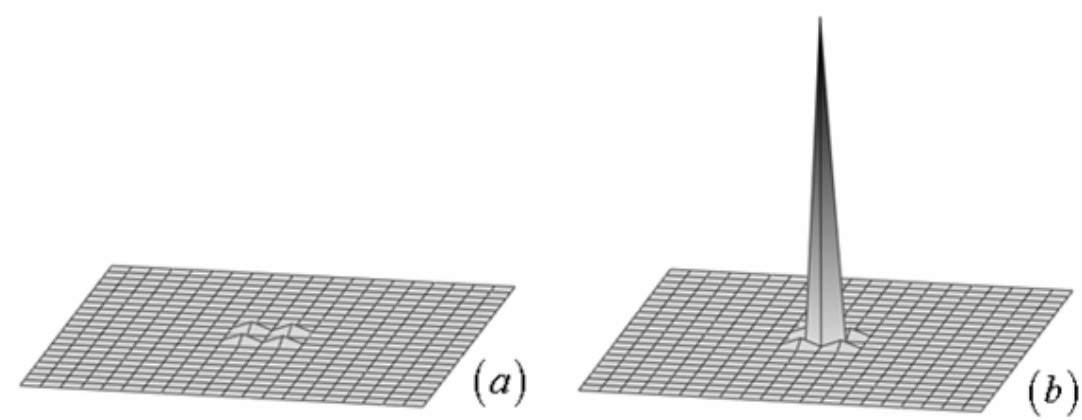

Figure 4: Logarithmic plot showing relative probabilities of energy transfer within an array with an aspect ratio of $1: 20$ (a) before; and (b) during application of laser radiation with $I=10^{14}$ $\mathrm{W} \mathrm{m}^{-2}$. The laser radiation ensures that energy will be transferred to the intended acceptor with a very high efficiency.

\section{DISCUSSION}

Until recently it appeared that only by inefficient and kinetically frustrated means, such as chromophore reorientation or movement, could significant control over resonant energy transfer be effected. The results presented here provide an insight into the way in which a beam of optical radiation can direct energy transfer through a near-field coupling mechanism. It has been shown that the throughput of laser light can facilitate a transfer that is otherwise completely 
forbidden, the system exhibiting a transmission that is switched on by a non-resonant beam. This kind of optical element effectively functions as an optical transistor - a fast and efficient, all-optical device that can mimic the functionality of its electronic counterpart. In view of the drive to scale down electronics components, such a device that operates on the molecular level should prove of considerable interest, particularly when it is scaled up into paired arrays. $^{13-15}$

Mechanisms for exerting and exploiting control over optical energy flow are only just beginning to receive due attention, ${ }^{14-20}$ and the challenge is to develop devices based on such principles. Our initial analysis has identified a concept that makes it possible to engineer a configuration of optical switches with parallel processing capability, without exhibiting significant cross-talk. Although the device presented demonstrates a high degree of efficiency, the next issue to address is the timescale over which it can function. While it is possible to exploit to advantage the variable delay that can be introduced between the initial excitation of donors and the subsequent transfer of their excitation (indeed this is the context for much current work on 'slow light') the excited state of the donors is not stable competing processes, including fluorescence and undesirable RET to peripheral acceptors, must be taken into account. The lifetime of the excited state will in a large part determine the lower threshold of the frequency with which energy transfer must be driven between the layers. Also, although RET between the donor and its counterpart acceptor is to be designedly forbidden, higher-order mechanisms for energy transfer need also be considered. These loss mechanisms can be guarded against by building fault-tolerance into the device network..$^{21,22}$

The next stage of development should resolve a number of practical considerations for the construction of arrays large enough to be implemented as electronics components, while retaining their functionality. Beyond the obvious demands for high brightness donors and efficient acceptors, it will be necessary, with orientational selectivity, to embed the donor and acceptor particles within a matrix that is transparent at the activating light frequencies. Numerous methods of nanoscale printing, many of which can easily achieve the necessary resolution, have been explored in the recent literature. Scanning probe lithography represents a general and sufficiently flexible technique offering the requisite precision; ${ }^{23}$ a well-proven and particularly promising method for molecular patterning and nanoimprinting is dip-pen nanolithography. ${ }^{24}$ The exact choice of method for printing and layering the donor and acceptor arrays will to some extent determine the optimum form of patterning for each array lattice, and it remains to be seen how this structure will be taken forward as an alternative to more conventional devices.

\section{ACKNOWLEDGEMENT}

RGC acknowledges a studentship from the Engineering and Physical Sciences Research Council, during which this research was undertaken.

\section{REFERENCES}

1. X. Hu, A. Damjanovik, T. Ritz and K. Schulten, "Architecture and mechanism of the light-harvesting apparatus of purple bacteria", Proc. Natl. Acad. Sci. USA, 95, pp. 5935-5941, 1998.

2. K. Mukai, S. Abe and H. Sumi, "Theory of rapid excitation-energy transfer from B800 to optically-forbidden exciton states of B850 in the antenna system LH2 of photosynthetic purple bacteria", J. Phys. Chem. B, 103, pp. 6096-6102, 1999.

3. P. Reineker, C. Supritz, C. Warns and I. Barvik, "Photosynthetic antennae systems: energy transport and optical absorption", J. Lumin., 108, pp. 149-152, 2004.

4. T. Brixner, J. Stenger, H. M. Vaswani, M. Cho, R. E. Blankenship and G. R. Fleming, "Two-dimensional spectroscopy of electronic couplings in photosynthesis", Nature, 434, pp. 625-628, 2005.

5. D. L. Andrews and A. A. Demidov, Resonance Energy Transfer (John Wiley \& Sons Ltd, Chichester, 1999).

6. G. J. Daniels, R. D. Jenkins, D. S. Bradshaw and D. L. Andrews, "Resonance energy transfer: The unified theory revisited", J. Chem. Phys., 119, pp. 2264-2274, 2003.

7. T. S. Forde and Q. S. Hanley, "Following FRET through five energy transfer steps: spectroscopic photobleaching, recovery of spectra, and a sequential mechanism of FRET", Photoch. Photobio. Sci., 4, pp. 609-616, 2005. 
8. D. P. Craig and T. Thirunamachandran, Molecular Quantum Electrodynamics: An Introduction to RadiationMolecule Interactions (Academic Press, London, 1984).

9. R. D. Jenkins, D. L. Andrews and L. C. Dávila Romero, "A new diagrammatic methodology for non-relativistic quantum electrodynamics", J. Phys. B: At. Mol. Opt. Phys., 35, pp. 445-468, 2002.

10. T. Förster, "Zwischenmolekulare Energiewanderung und Fluoreszenz", Ann. Phys.-Berlin, 2, pp. 55-75, 1948.

11. D. P. Craig and T. Thirunamachandran, "Radiation-molecule and molecule-molecule interactions - a unified viewpoint from quantum electrodynamics", Accounts Chem. Res., 19, pp. 10-16, 1986.

12. P. Allcock, R. D. Jenkins and D. L. Andrews, "Laser-assisted resonance-energy transfer", Phys. Rev. A, 6102, 023812, 2000.

13. D. L. Andrews and R. G. Crisp, "Optically-activated energy transfer: array implementation", J. Opt. A: Pure Appl. Opt., 8, pp. S106-S112, 2006.

14. R. G. Crisp and D. L. Andrews, "Optical switching in nano-arrays: transistor action through directed energy transfer", in Nanophotonics, Proc. SPIE 6195, 61951I, 2006.

15. D. L. Andrews and R. G. Crisp, "Theory of directed electronic energy transfer", J. Fluor. 16, pp. 191-199, 2006.

16. B. S. Ham, "A novel method of all-optical switching: Quantum router", Etri J. 23, pp. 106-110, 2001.

17. M. A. J. Klik, T. Gregorkiewicz, I. V. Bradley and J. P. R. Wells, "Optically induced deexcitation of rare-earth ions in a semiconductor matrix", Phys. Rev. Letts 89, 227401, 2002.

18. T. Kawazoe, K. Kobayashi, S. Sangu and M. Ohtsu, "Demonstration of a nanophotonic switching operation by optical near-field energy transfer", Appl. Phys. Letts 82, pp. 2957-2959, 2003.

19. O. Wada, "Femtosecond all-optical devices for ultrafast communication and signal processing", New J. Phys. 6, 183, 2004.

20. M. Naruse, T. Kawazoe, S. Sangu, K. Kobayashi and M. Ohtsu, "Optical interconnects based on optical far- and near-field interactions for high-density data broadcasting", Opt. Express 14, pp. 306-313, 2006.

21. C. C. Fan and J. Bruck, "Tolerating multiple faults in multistage interconnection networks with minimal extra stages", IEEE T. Comput., 49, pp. 998-1004, 2000.

22. M. Choi, N. Park and F. Lombardi, "Modeling and analysis of fault tolerant multistage interconnection networks", Ieee T. Instrum. Meas., 52, pp. 1509-1519, 2003.

23. B. D. Gates, Q. B. Xu, M. Stewart, D. Ryan, C. G. Willson and G. M. Whitesides, "New approaches to nanofabrication: Molding, printing, and other techniques", Chem. Rev., 105, pp. 1171-1196, 2005.

24. A. Mulder, S. Onclin, M. Peter, J. P. Hoogenboom, H. Beijleveld, J. ter Maat, M. F. Garcia-Parajo, B. J. Ravoo, J. Huskens, N. F. van Hulst and D. N. Reinhoudt, "Molecular printboards on silicon oxide: Lithographic patterning of cyclodextrin monolayers with multivalent, fluorescent guest molecules", Small, 1, pp. 242-253, 2005 